\title{
Systematic studies on Cucurbitaceae family at Rajshahi division, Bangladesh
}

\author{
A H M Mahbubur Rahman \\ Department of Botany, University of Rajshahi, Rajshahi-6205, Bangladesh
}

Email address:

ahmmahbubur_rahman@yahoo.com

\section{To cite this article:}

A H M M Rahman. Systematic Studies on Cucurbitaceae Family at Rajshahi Division, Bangladesh. Plant. Vol. 1, No. 2, 2013 , pp. 10-15. doi: $10.11648 /$ j.plant.20130102.11

\begin{abstract}
Systematic investigation on the family Cucurbitaceae growing throughout the Rajshahi Division situated in the northern parts of Bangladesh was carried out. A total of 24 species under 13 genera of the family Cucurbitaceae were collected and identified. A complete taxonomic account of each species has been given along with their current name, local name, brief description, phenology, basic chromosome number and voucher number. A new system of classification of the family Cucurbitaceae has been proposed on the basis of morphological, cytological and floral characters.
\end{abstract}

Keywords: Systematic Studies, Cucurbitaceae, Rajshahi Division, Bangladesh

\section{Introduction}

Cucurbitaceae is a medium sized and botanically highly specialized family of mainly climbing plants. Cucurbitaceae is a fairly large family containing about 100 genera and 800 species which are mainly tropical or subtropical in distribution, with a few species extending into temperate climate [18]. The cultivated species investigated in this study belong to the genera Benincasa, Citrullus, Cucumis, Cucurbita, Lagenaria, Luffa, Momordica and Trichosanthes. These are used as fruits and vegetables, and most of them have considerable economic value[15].

Many species of this family are the sources of medicine. Some are widely cultivated in the field for vegetable and food. A few taxa are also grown as ornamental plants. Cucurbitaceae is the largest group of summer vegetable crops. These include cucumber, musk melon, water melon, tinda, bottle gourd, luffa, and bitter gourd, pumpkin, squashes, parwal and snake gourd. Most of the members of the family Cucurbitaceae are monoecious and a few are dioecious. A number of hermaphrodite and andromonoecious cultivars are also available in some crops. They are all summer season crops and are susceptible to frost [3].

\section{Materials and Methods}

Systematic investigation on the family Cucurbitaceae growing throughout the Rajshahi division, Bangladesh was carried out. The study area of Rajshahi division includes six districts: Rajshahi (including three thanas Boalia, Godagari, Tanore), Nawabgonj (including three thanas Sadar, Nachole, Sibgonj), Naogaon (including two thanas Atrai, Raninagar), Joypurhat (including two thanas Akkelpur, Khetlal), Bogra (including two thanas Adamdighi, Dupchachia) and Natore (including three thanas Sadar, Singra, Lalpur). A total of 24 species under 13 genera of the family Cucurbitaceae were collected and identified.

A survey on the determination of the location of different species was made and a list was prepared to be acquainted with the Cucurbitaceae available in the selected area. All the species were noted and from time to time the areas were visited to see when they flowered. For the morphological study, different types of species were examined again and again in order to see if there was any variation or not. They were collected at flowering stages and herbarium specimens were prepared as vouchers. In this practice standard method was followed. In this regard different types of plant species were collected from different habitats. All the collected plant specimens were kept in the Herbarium, Department of Botany, and University of Rajshahi, Bangladesh.

The collected specimens were identified by referring to the related taxonomic books and booklets from the library of Rajshahi University. The major collected materials were identified and described up to species with the help of [7], [14], [4], [10] and [17]. In some cases [11], [6], [16], [2], [1] were consulted. For the current name and up to date nomenclature [8], [9], [12] and [13] were also consulted. 


\section{Results and Discussion}

Systematic investigation on the family Cucurbitaceae growing throughout the Rajshahi Division situated in the northern parts of Bangladesh was carried out. A total of 24 species under 13 genera of the family Cucurbitaceae were collected and identified. A complete taxonomic account of each species has been given along with their current name, local name, brief description, phenology, basic chromosome number and voucher number.

Most of the cultivated species were grown in all districts of Bangladesh. Some of the species which were sometimes cultivated and sometimes wild were grown in particular area. Most of the species were distributed all over the study area, i.e. Benincasa hispida, Citrullus lanatus, Coccinia grandis, Cucumis melo, Cucumis sativus, Cucurbita maxima, Cucurbita moschata, Cucurbita pepo, Lagenaria siseraria, Luffa acutangula, Luffa cylindrica, Momordica charantia, Momordica cochinchinensis, Melothria maderaspatana, Trichosanthes anguina, Trichosanthes cucumerina, Trichosanthes bracteata and Trichosanthes dioica.

One species growing in one district of the study area, i.e. Thladiantha cordifolia, populated at Godagariin Rajshahi roadside of bushes. Gymnopetalum cochinchinense and Diplocyclos palmatus were very rare species in the study area. Momordica dioica was collected from Rajshahi and Joypurhat districts. Cucumis callosus was collected from Naogaon and Joypurhat districts. Trichosanthes cordata was collected from Rajshahi and Natore districts. Gymnopetalum cochinchinense was collected from Rajshahi district.

On the basis of morphological, cytological and floral characteristics a new system of classification of the family Cucurbitaceae has been proposed as follows:

Tribe-1: Benincaseae-Ovule many horizontal, fruit usually smooth or indehiscent. i.e. Benincasa, Lagenaria, Luffa.

Tribe-2: Cucurbiteae- Stamens 5, apparently 3, anther lobes sigmoid S or U-shaped. Ovules erect or horizontal. Fruit fleshy, indeiscent and 1to many seeded. i.e. Cucurbita.

Tribe-3: Cucumerinae-Ovule horizontal, stamens free. i.e. Cucumis, Citrullus, Coccinea, Diplocyclos, Gymnopetalum.

Tribe-4: Jolifeieae-Petals fringed or with ventral scales. i.e. Momordica.

Tribe-5: Melothrieae-Stamens usually 5, but apparently 3, rarely 2 or 4 , anther lobes straight or slightly curved. i.e. Melothria.

Tribe-6: Thladiantheae: Stamens free or only connate at base, 5 rarely 4. i.e. Thladiantha.

Tribe-7: Trichosantheae-Petals entire or with fimbricate margin. Ovules horizontal. Fruit fleshy or dry, dehiscing by three valves. i.e. Trichosanthes.

By examining the plant materials collected from the study area using the identification methods, systematic information was accumulated and described below.

\subsection{Botanical Name: Benincasa hispida (Thunb.) Cogn}

Brief description: A large, softly hairy climber, tendrils long, 3-branched. Leaves cordate, reniform, orbicular, 7-8 lobed, petiole length $12.5 \mathrm{~cm}$., without glands. Flowers large, yellow, monoecious, all solitary, sepals 5, free, petals 5, obovate, slightly connate. Stamens 3, anthers exserted, 2 large, carpel 3, fruit large, covered with white waxy substances, length $30 \mathrm{~cm}$. breadth $36 \mathrm{~cm}$.

Local Name: Chal kumra, sada kumra, chuna kumra

Phenology: March to October

Chromosome Number: $2 \mathrm{n}=24$ [19]

Specimens examined: MR 01 Boalia, Rajshahi 10-05-2001; MR 147 Sadar, Nawabgonj 10-04-2002; MR 06 Sadar, Natore 03-06-2001; MR 07 Dupchachia, Bogra 15-06-2001; MR 08 Atrai, Naogaon 17-06-2001; MR 233 Akkelpur, Joypurhat 10-06-2003.

\subsection{Botanical Name: Citrullus lanatus (Thunb.) Mart \& Nakai}

Brief description: Climbing herbs, stems are angular villose. Leaves are triangular ovate, cordate, deeply individed, glabrous and hardly scabrid. Flowers are yellow. Male flower-peduncles elongate, vilous, calyx-tube broadly campanulate, corolla greenish, villose. Female flower-calyx and corolla as in the male, ovary long. Fruits subglobose or ellipsoid, smooth, flesh rose to red, pulp sweet, red or yellow. Seeds are ovate, black or red.

Local Name: Turmuz

Phenology: January to May

Chromosome Number: $2 \mathrm{n}=22$ [19]

Specimens examined: MR 13 Tanore, Rajshahi 03-09-2001; MR 14 Sibgonj, Nawabgonj 15-09-2001; MR 225 Singra, Natore 01-03-2003; MR 149 Adamdighi, Bogra 20-03-2003; MR 15 Atrai, Naogaon 17-09-2001; MR 16 Khetlal, Joypurhat 18-09-2001.

\subsection{Botanical Name: Coccinea grandis (L.) Voigt.}

Brief description: A climbing herb, stems are much branched, glabrous or white scaly, petiole and tendrils are slender. Flowers are white. Male flowers-peduncle jointed below the flowers. Calyx-tube linear oblong, corolla white, triangular. Female flower-peduncles slender, staminodes 3, ovary smooth, glabrous, stigma densely papilose. Fruits fusiform ovoid, cylindrical, smooth, bright scarlet. Seeds oblong-ovoid, smooth.

Local Name: Telackucha

Phenology: March to December

Chromosome Number: $2 \mathrm{n}=24$ [5]

Specimens examined: MR 151 Boalia, Rajshahi 16-05-2002; MR 21 Sadar, Nawabgonj 12-10-2001; MR 22 Sadar, Natore 15-10-2001; MR 23 Dupchachia, Bogra 18-10-2001; MR 227 Atrai, Naogaon 14-04-2003; MR 152 Akkelpur, Joypurhat 15-06-2002.

\subsection{Botanical Name: Cucumis sativus $\mathbf{L}$.}

Brief description: Climbing herbs, stem scabrous. Leaves 5 -angualr or slightly lobed, lobes acute hispidulous on both surface and also often with soft hairs. Leaves 7.5-12.5 cm., 
petiole $5-7.5 \mathrm{~cm}$. petals $1.5 \mathrm{~cm}$., female peduncle sometimes $5 \mathrm{~cm}$., young ovary muricate with rigid prickles. Fruit glabrous sometimes tuberculated commonly elongated, cylindric 30.5 by $3.7 \mathrm{~cm}$. the cucumber.

Local Name: Sasha, Khira

Phenology: January to December

Chromosome Number: $2 \mathrm{n}=14$ [19]

Specimens examined: MR 24 Boalia, Rajshahi 20-10-2001; MR 25 Sibgonj, Nawabgonj 22-10-2001; MR 26 Singra, Natore 29-10-2001; MR 27 Dupchachia, Bogra 31-10-2001; MR 153 Atrai, Naogaon 07-05-2002; MR 154 Akkelpur, Joypurhat 30-06-2002.

\subsection{Botanical Name: Cucumis melo L.}

Brief description: Stems are scabrous, leaves are orbicular reniform 5-angular or lobed lobes neither deep nor acute scabrid on both surfaces and also often with soft hairs. Female peduncle sometimes $5 \mathrm{~cm}$. petals $4.06 \mathrm{~cm}$. Fruit glabrous of somewhat hairy not spinous nor tuberculate, spherical ovoid elongate or contorted.

Local Name: Phuti, Bangi, Kurbuz

Phenology: January to May

Chromosome Number: 2n=24 [19]

Specimens examined: MR 157 Tanore, Rajshahi 10-03-2002; MR 66 Sibgonj, Nawabgonj 12-03-2002; MR 37 Sadar, Natore 29-11-2001; MR 70 Dupchachia, Bogra 11-04-2002; MR 38 Atrai, Naogaon 07-12-2001; MR 228 Akkelpur, Joypurhat 13-06-2003.

\subsection{Botanical Name: Cucumis callosus L.}

Brief description: Stem slender, angular, hirsute. Petioles slender, hispid, lamina cordate, suborbicular, deeply palmately 5-7 lobed, scabrid. Tendril simple. Male flower-generally solitary long peduncled, calyx-tube narrow campanulate, corolla yellow, oblong, acute, stamina filament short. Female flower-ovary densely hairy, stigmas converging. Fruit smooth, obovoid, seeds oblong, white.

Local Name: Bangumak

Phenology: June to October

Chromosome Number: $2 \mathrm{n}=24$ [19]

Specimens examined: MR 233 Atrai, Naogaon 17-03-2003; MR 234 Akkelpur, Joypurhat 09-06-2003.

\subsection{Botanical Name: Cucurbita maxima Duch}

Brief description: Annual, with long trailing, soft stems, more or less round in cross section. Leaves large, dark-green and cordate. Male flower-It carried on long, cylindrical peduncles, sepals 5, petals 5, stamens 3 , anthers 3 , fused. Female flower-it is larger than the males, short peduncles, petals 5 , carpels 3 , style short, stigmas 3 , fruit variable in size and colour, round, flattened or oval.

Local Name: Bitati, mistikumra

Phenology: January to December

Chromosome Number: $2 \mathrm{n}=40$ [19]

Specimens examined: MR 167 Boalia, Rajshahi 10-03-2002; MR 168 Sadar, Nawabgonj 12-03-2002; MR 54
Lalpur, Natore 09-03-2001; MR 55 Adamdighi, Bogra 11-04-2001; MR 56 Atrai, Naogaon 07-05-2001; MR 169 Akkelpur, Joypurhat 13-06-2002.

\subsection{Botanical Name: Cucurbita pepo L.}

Brief description: Annual, with a long trailing, angular, prickly stem and large roughly triangular leaves, 5-lobed sinus between the lobes broad. Fruiting peduncle woody strongly grooved. Male peduncle $10 \mathrm{~cm}$. or more, female 3.2 $\mathrm{cm}$. corolla $5-10 \mathrm{~cm}$. Fruits vary greatly in size, colour and shape among different cultivars, some being long, round and smooth, while others are flattened or more or less spherical.

Local Name: Mistikadu, Bilati

Phenology: November to June

Chromosome Number: $2 \mathrm{n}=40$ [19]

Specimens examined: MR 273 Boalia, Rajshahi 06-10-2003; MR 61 Sibgonj, Nawabgonj 09-03-2001; MR 276 Sadar, Natore 20-10-2003; MR 171 Dupchachia, Bogra 11-04-2002; MR 62 Raninagar, Naogaon 07-05-2001; MR 236 Akkelpur, Joypurhat 13-06-2003.

\subsection{Botanical Name: Cucurbita moschata (Duch. ex Lam.) Duch}

Brief description: Annual with long trailing, soft stems without prickly hairs and palmately divided lobes. Fruit variable, usually large, globular, cylindrical, seeds with a thin or ragged margin, scalloped or shredded in appearance, the margin more deeply coloured than the body of the seed, which is $16-20 \mathrm{~mm}$. long.

Local Name: Mistikadu, Bilati

Phenology: November to June

Chromosome Number: $2 \mathrm{n}=40$ [19]

Specimens examined: MR 63 Singra, Natore 09-03-2001; MR 274 Boalia, Rajshahi 15-10-2003; MR 277 Sadar, Nawabgonj 20-10-2003;; MR 172 Dupchachia, Bogra 11-04-2002; MR 64 Atrai, Naogaon 07-05-2001; MR 237 Khetlal, Joypurhat 13-06-2003.

\subsection{Botanical Name: Diplocyclos palmatus (L.) Jeffrey}

Brief description: Stem slender, much branched, glabrous. Petioles 2-6 cm. long, lamina suborbicular in outline, 3-lobed, lobes elliptic acuminate, base cordate. Flowers white or yellowish. Male flower-calyx-tube broadly campanulate, corolla campanulate, stamens 3, anther loculi, linear, slightly flexuous. Female flower-calyx and corolla as in male, staminodes 3, ovary subglobose, with 3-placentas, stigma 3, fruit globose or ovoid, seeds ovate.

Local Name: Mala

Phenology: November to April

Chromosome Number: $2 \mathrm{n}=24$ [5]

Specimens examined: MR 174 Godagari, Rajshahi 10-03-2002; MR 67 Sadar, Nawabgonj 12-09-2001.

\subsection{Botanical Name: Gymnopetalum cochinchinense (Lour.) Kurj}

Brief description: Stems scandent, petioles slender, 
lamina ovate, swallowly 3-lobed, acuminate, base deeply cordate, margin toothed. Flowers white. Male flowersolitary or in racemes of 3-8 flowers, bracts ovate-oblong, deeply toothed, calyx tube subcylindric, corolla lobes white, oblong-ovate, anthers both ends acute. Female flower-peduncle 1-4 cm. long, fruits ovoid-oblong, ribbed.

Local Name: Kaubuti

Phenology: November to April

Chromosome Number: $2 \mathrm{n}=22$ [5]

Specimens examined: MR 68 Godagari, Rajshahi 10-03-2001.

\subsection{Botanical Name: Lagenaria siceraria (Molina) Standl}

Brief description: Pubescent, tendrils 2-fid. Leaves ovate or orbicular, cordate, dentate, petiole long, with two glands at it apex. Flowers large, white, solitary, monoecious or dioecious, the males long, the females short, petals 5, free, obovate, stamens 3, anthers connate. Female flower-calyx and corolla as in the male, ovary oblong, ovules many, horizontal, placentas 3, vertical. Fruit large, ultimately thick membranous or almost woody, indehiscent. Seeds many, horizontal, compressed, smooth.

Local Name: Panilau, Lau, Panikadu

Phenology: January to December

Chromosome Number: $2 \mathrm{n}=22$ [19]

Specimens examined: MR 180 Boalia, Rajshahi 10-03-2002; MR 130 Sibgonj, Nawabgonj 12-03-2003; MR 78 Sadar, Natore 19-03-2001; MR 79 Dupchachia, Bogra 11-04-2001; MR 80 Raninagar, Naogaon 07-05-2001; MR 242 Akkelpur, Joypurhat 13-06-2003.

\subsection{Botanical Name: Luffa acutangula (L.) Roxb.}

Brief description: Stout monoecious climber, foetid and when bruised. Leaves 5 angled or swallowly lobed, pale green beneath, scabrous, $10-25 \mathrm{~cm}$. Male flower in racemes of several flowers; female flower solitary, ovary inferior, filiform, with 10 longitudinal ribs on which are swollen glands, style short, stigmas 3. Fruit elongate, club-shaped, crowned by enlarged sepals and style angled, 10 ribbed, many seeded.

Local Name: Toroy, Jhinga

Phenology: June to October

Chromosome Number: $2 \mathrm{n}=26$ [19]

Specimens examined: MR 104 Godagari, Rajshahi 10-03-2001; MR 197 Sibgonj, Nawabgonj 12-03-2002; MR 108 Sadar, Natore 29-03-2001; MR 109 Dupchachia, Bogra 11-04-2001; MR 110 Raninagar, Naogaon 07-05-2001; MR 249 Akkelpur, Joypurhat 13-06-2003.

\subsection{Botanical Name: Luffa cylindrica (L.) Roem}

Brief description: Extensively climbing hairy, stem 5-angled tendrils 2-3 fid or more. Leaves $10 \mathrm{~cm}$. reniform, orbicular, 5-angled, dentate. Male peduncle $15 \mathrm{~cm}$., flowers often approximate near the summit, petals 5, yellow, stamens 5. Female flowers solitary, fruit elongate clavate smooth 10-ribbed or somewhat 10-angular, seed usually black, very narrowly winged, smooth or very sparingly tubercled.

Local Name: Dhundol

Phenology: June to November

Chromosome Number: 2n=26 [19]

Specimens examined: MR 111 Tanore, Rajshahi 10-03-2001; MR 112 Nachole, Nawabgonj 12-03-2001; MR 198 Singra, Natore 09-03-2002; MR 113 Dupchachia, Bogra 11-04-2001; MR 199 Raninagar, Naogaon 07-05-2002; MR 250 Akkelpur, Joypurhat 13-06-2003.

\subsection{Botanical Name: Melothria maderaspatana (L.) Cogn}

Brief description: Stems are slender, angular hispid or scabrous. Petioles are filiform, lamina 3-5 lobed, acute or subacuminate, tendrils slender. Male flower in cluster, peduncle short, calyx narrow, corolla hairy outside connective of stamens produced. Female flowers- solitary or subfascicled, fruiting peduncles almost absent, calyx hairy. Fruit globes, red and glossy at maturity, seeds ovoid, grey, turgid.

Local Name: Agmuki

Phenology: November to April

Chromosome Number: $2 \mathrm{n}=22$ [5]

Specimens examined: MR 117 Boalia, Rajshahi 10-03-2001; MR 202 Sadar, Nawabgonj 12-03-2002; MR 203 Sadar, Natore 29-03-2002; MR 118 Dupchachia, Bogra 11-04-2001; MR 119 Raninagar, Naogaon 07-05-2002; MR 252 Akkelpur, Joypurhat 13-06-2002.

\subsection{Botanical Name: Momordica cochinchinensis (Lour.) Spreng}

Brief description: Dioecious, leaves 10-12.5 cm. cordate ovate, usually 3-lobed, glabrous or a little pubescent, petioles glandular, flower large white, petals $2.5-5 \mathrm{~cm}$., white tinged with yellow, 3 with black spots at the base 2 with yellow glands. Female peduncle $2.5-5 \mathrm{~cm}$. bract small about the middle. Fruit muricated, ovate, pointed, very fleshy, horizontal, ovate, compressed.

Local Name: Kakrol

Phenology: March to September

Chromosome Number: $2 \mathrm{n}=22$ [19]

Specimens examined: MR 120 Tanore, Rajshahi 10-03-2001; MR 204 Sibgonj, Nawabgonj 12-03-2002; MR 121 Lalpur, Natore 19-03-2002; MR 122 Dupchachia, Bogra 11-04-2002; MR 205 Raninagar, Naogaon 07-05-2002; MR 253 Akkelpur, Joypurhat 13-06-2001.

\subsection{Botanical Name: Momordica charantia L.}

Brief description: Monoecious, bract about the middle of the male peduncle orbicular entire, leaves $2.5-7.5 \mathrm{~cm}$., orbicular glabrous or slightly pubescent. Male peduncle 5-10 cm., slender, calyx-lobes ovate, acute. Petals 1-2 cm. yellow. Female peduncle $5-10 \mathrm{~cm}$., slender, bracteates near the base, ovary fusiform, muricate. Fruit ovoid narrow to 
both ends many-ribbed covered with triangular tubercles. Seeds compressed.

Local Name: Karala

Phenology: January to December

Chromosome Number: $2 \mathrm{n}=22$ [19]

Specimens examined: MR 123 Boalia, Rajshahi 10-03-2001; MR 124 Sibgonj, Nawabgonj 12-03-2001; MR 206 Lalpur, Natore 09-03-2002; MR 125 Adamdighi, Bogra 11-04-2001; MR 210 Raninagar, Naogaon 07-05-2003; MR 254 Akkelpur, Joypurhat 13-06-2002.

\subsection{Botanical Name: Momordica dioica Roxb.}

Brief description: Stems are slender, petioles 1-2 cm. long, lamina ovate, acuminate, base cordate, glabrous. Tendrils filiform, simple, flowers yellow. Male flower- solitary, peduncles slender, bracteate at apex, corolla lobes yellow, oblong, veins black grey. Female flowers- peduncles ebracteate, bract small, near the base, ovary ovoid, oblong, densely coarse-hairy. Fruits ovoid, densely soft echinate, seeds are pale-yellow, broadly ovoid.

Local Name: Gheekorolla

Phenology: June to October

Chromosome Number: $2 \mathrm{n}=22$ [19]

Specimens examined: MR 271 Boalia, Rajshahi 15-09-2003; MR 131 Akkelpur, Joypurhat 13-06-2001.

\subsection{Botanical Name: Thladiantha cordifolia (B1.) Cogn}

Brief description: Stems are sulcate, pubescent, petiole slender, villose, lamina ovate, acuminate, base deeply cordate rounded. Tendrils simple, flower golden yellow. Male flowers-many, approximate at the summit of long peduncle, calyx lobes linear oblong, corolla yellow, lobes ovate, stamens 5, anther one celled, straight. Female flowers-peduncles one flowered, slender, villous, ovary oblong, villose, stigma reniform, fruit oblong, seeds ovoid.

Local Name: Dabilata

Phenology: May to October

Chromosome Number: $2 \mathrm{n}=18$ [5]

Specimens examined: MR 132 Godagari, Rajshahi 13-06-01.

\subsection{Botanical Name: Trichosanthes cordata Roxb.}

Brief description: An extensive climber, dioecious, leaves often $15-20 \mathrm{~cm}$. acute or obscurely angular-lobed hair beneath, bracts elongate obovate entire or scarely serrate, tendrils usually 3 fid. Male peduncles usually paired, the racemed one often $20 \mathrm{~cm}$. stout, bracts pubescent, calyx-tube $3.5 \mathrm{~cm}$. densely hairy without segments finely acuminate. Fruit globose, size of small orange, seeds 1-2 cm. scarcely angular on the margin.

Local Name: Bhuikakra

Phenology: May to October

Chromosome Pumber: 2n=22 [19]

Specimens examined: MR 132 Godagari, Rajshahi 13-06-01; MR 214 Sadar, Natore 09-03-2002.

\subsection{Botanical Name: Trichosanthes dioica Roxb.}

Brief description: Stems twining extensively, more or less wooly and scabrous, dioecious. Leaves cordate, oblong, acute, petiole scabrous wooly, tendrils 2-fid. Male flowersnot racemed woolly without, anthers free. Male peduncles paired, the second-flowering carrying a raceme of about 12 flowers. Calyx-tube $3.8 \mathrm{~cm}$. narrow. Fruit $8.9 \mathrm{~cm}$., oblong or nearly spherical, acute, orange-red. Seeds half-ellipsoid, compressed, corrugateon the margin.

Local Name: Potol

Phenology: November to April

Chromosome Number: $2 \mathrm{n}=22$ [19]

Specimens examined: MR 215 Boalia, Rajshahi 15-03-2002; MR 261 Sadar, Nawabgonj 15-07-2003; MR 134 Sadar, Natore 09-03-2001; MR 260 Adamdighi, Bogra 30-06-2003; MR 262 Raninagar, Naogaon 20-07-2003; MR 259 Akkelpur, Joypurhat 17-06-2003.

\subsection{Botanical Name: Trichosanthes anguina $\mathrm{L}$.}

Brief description: Monoecious climbing annual herb, 5-angled, furrowed, tendrils branched. Leaves cordate subreniform 5 lobed or 5-angular lobes, not acuminate pubescent or puberulous on both surface, fruit elongate cylindric sometimes contorted, seeds corrugate.

Local Name: Chichinga

Phenology: November to April

Chromosome Number: 2n=22 [19]

Specimens examined: MR 217 Adamdighi, Bogra 11-04-2002; MR 136 Akkelpur, Joypurhat 02-03-2001; MR 263 Godagari, Rajshahi 25-07-2003; MR 265 Nachole, Nawabgonj 18-08-2003; MR 264 Lalpur, Natore 01-08-2003;; MR 266 Raninagar, Naogaon 15-08-2003.

\subsection{Botanical Name: Trichosanthes bracteata (Lamk.) Voigt}

Brief description: Stems are woody below, robust, stout branching sulcate, pubescent. Petioles striate, lamina broadly ovate or suborbicular. Tendrils 3-fid, flowers white. Male flower-racemed, peduncles robust, apex 5-10 flowered, calyx-tube longitudinally striate, acuminate. Female flower-axillary, solitary, calyx-tube segment,acuminate, style long. Fruit globose, not acute, seeds ovate oblong, embedded in black pulp.

Local Name: Makal, Makalphal

Phenology: July to December

Chromosome Number: $2 \mathrm{n}=22$ [19]

Specimens examined: MR 219 Adamdighi, Bogra 11-04-2002; MR 256 Akkelpur, Joypurhat 13-06-2003; MR 218 Tanore, Rajshahi 10-03-2002; MR 139 Nachole, Nawabgonj 12-03-2001; MR 140 Sadar, Natore 19-03-2001;; MR 141 Raninagar, Naogaon 07-05-2001.

\subsection{Botanical Name: Trichosanthes cucumerina L.}

Brief description: Stema are slender glabrous or slightly hairy. Petioles 2-7 cm. long, lamina suborbicular reniform or 
broadly ovate. Tendrils 2-3 fid, flowers white. Male flowers-peduncles in pairs, the earliar 1-flowered, calux-tube narrowed to the base, anthers oblong. Female flowers-calyx-tube gradually dilated upwards, lower part often hairy, ovary oblong, style filiform. Fruits are ovoid, conica, smooth, seeds are ovate oblong.

Local Name: Banchichinga

Phenology: June to November

Chromosome Number: $2 \mathrm{n}=22$ [19]

Specimens examined: MR 142 Boalia, Rajshahi 10-03-2001; MR 220 Sibgonj, Nawabgonj 12-03-2002; MR 143 Singra, Natore 29-03-2001; MR 221 Adamdighi, Bogra 11-04-2002; MR 144 Atrai, Naogaon 07-05-2001; MR 257 Khetlal, Joypurhat 13-06-2003.

\section{Conclusion}

Systematic investigation on the family Cucurbitaceae growing throughout the Rajshahi Division situated in the northern parts of Bangladesh was carried out. A total of 24 species under 13 genera of the family Cucurbitaceae were collected and identified. A new system of classification of the family Cucurbitaceae has been proposed on the basis of morphological, cytological and floral characters.

\section{Acknowledgements}

The author is grateful to the Ministry of Science, Information and Communication Technology, Government People's Republic of Bangladesh for financial support to complete this research work.

\section{References}

[1] Ahmed Z U, Begum Z N T, Hassan M A, Khondker M, Kabir S M H, Ahmad M, Ahmed A T A,Rahman A K A and Haque E U(Eds). Encyclopedia of Flora and Fauna of Bangladesh. Angiosperms; Dicotyledons.Asiatic Society ofBangladesh, Dhaka., Vol 8, 2009.

[2] BhattacharyyaB and Johri B M. Flowering Plants Taxonomy and Phylogeny. Prokas Publishers, Calcutta, India., 1998; pp.375-378.

[3] Choudhury B. Vegetables. National Book Trust, New Delhi, India, 1967; pp.130-162.
[4] Cronquist A. The Evolution and Classification of Flowering Plants. Houghton Miftlin, Boston. U.S.A., 1968.

[5] Darlington CD, Wylie AP. Chromosome Atlas of Flowering Plants. George Allen and Unwin Ltd. Ruskin House Museum Street, London, U.K., 1950; pp.98-100.

[6] Heywood V H. Flowering Plants of the World. Oxford University Press, New York, U.S.A., 1979; pp.115-117.

[7] Hooker J D. Flora of British India. L. Reeve and Co. Ltd. London Vol 2, 1961; pp.604-635.

[8] Huq A M. Name Changes in Bangladesh Angiosperms. Bangladesh National Herbarium, BARC, Dhaka, Bangladesh., 1986; pp.1-104.

[9] Huq A M. Plant Names of Bangladesh. Bangladesh National Herbarium, BARC, Dhaka, Bangladesh., 1986; pp.1-289.

[10] Kirtikar K R andBasu B D. Indian Medicinal Plants. Lalit Mohan Basu, Allahabad, Jayyed Press, New Delhi, India., Vol 2, 1987; pp.1104-1169.

[11] Lawrence G H M. Taxonomy of Vascular Plants. Oxford and IBM Publishing Co., Rakes Press, New Delhi, India., 1973; pp.718-720.

[12] Pasha M K and Zaman M B. Name Changes in Plants of Bangladesh. Chittagong University Studies, Part-II, Science Vol. 12(1), 1988; 107-112.

[13] Pasha M K and Uddin S B. Dictionary of Plant Names of Bangladesh. Janokalyan Prokashani, Chittagong-4000, Bangladesh., 2013; pp.1-434.

[14] Prain D.Bengal Plants. Botanical Survey of India. Calcutta, India. Vol 1, 1963; pp.374-385.

[15] Purseglove JW. Tropical Crops Dicotyledons. Longman Group Limited. London., 1968; pp.108-138.

[16] Rahman, A H M M, Islam AKMR and Hossain M M. Taxonomy of Cucurbitaceae: Taxonomic investigation of wild \& cultivated cucurbits of Northern Parts of Bangladesh. LAP Lambert publishing, Germany, 2013; pp.1-176.

[17] Sharma OP. Plant Taxonomy. Tata McGrow-Hill Publishing Company Limited. New Delhi. India., 2004; pp.18-42.

[18] Subrahmanyam NS. Modern Plant Taxonomy. Vikas Publishing House Pvt. Ltd. New Delhi, India, 2004; pp.316-321.

[19] Simmonds NW. Evolution of Crop Plants. Longman Group Ltd. London, 1976. 